\title{
The Promoted Generation of Basal Forebrain Cholinergic Neurons from Neural Progenitor Cells on Three-dimensional Graphene foam Scaffold
}

\section{Ziyun Jiang}

Suzhou Institute of Nano-tech and Nano-Bionics Chinese Academy of Sciences: Chinese Academy of

Sciences Suzhou Institute of Nano-tech and Nano-Bionics

\section{Lingyan Yang}

Suzhou Institute of Nano-tech and Nano-Bionics Chinese Academy of Sciences: Chinese Academy of

Sciences Suzhou Institute of Nano-tech and Nano-Bionics

\section{Linhong Zhou}

Suzhou Institute of Nano-tech and Nano-Bionics Chinese Academy of Sciences: Chinese Academy of

Sciences Suzhou Institute of Nano-tech and Nano-Bionics

\section{Miao Xiao}

Suzhou Institute of Nano-tech and Nano-Bionics Chinese Academy of Sciences: Chinese Academy of

Sciences Suzhou Institute of Nano-tech and Nano-Bionics

\section{Sancheng $\mathrm{Ma}$}

Suzhou Kowloon Hospital Shanghai Jiao Tong University School of Medicine

Wei Jin

Nanjing Drum Tower Hospital: Nanjing University Medical School Affiliated Nanjing Drum Tower Hospital

\section{Guosheng Cheng ( $\nabla$ gscheng2006@sinano.ac.cn )}

Suzhou Institute of Nano-tech and Nano-Bionics Chinese Academy of Sciences https://orcid.org/00000003-4810-9403

\section{Research}

Keywords: Basal forebrain cholinergic neurons, Alzheimer's disease, 3D-GF, Neural progenitor cells, Differentiation

Posted Date: September 8th, 2021

DOI: https://doi.org/10.21203/rs.3.rs-864686/v1

License: (c) (1) This work is licensed under a Creative Commons Attribution 4.0 International License. Read Full License 


\section{Abstract}

Background: An early substantial loss of basal forebrain cholinergic neurons (BFCNs) is a common property of Alzheimer's disease and the generation of functional BFCNs is related to learning and memory deficits. As a biocompatible and conductive scaffold for growth of neural stem cells, three-dimensional graphene foam (3D-GF) supports applications in tissue engineering and regenerative medicine. Although its effects on differentiation have been demonstrated, the effect of 3D-GF scaffold on the generation of BFCNs still remains unknown.

Methods: In this study, we used 3D-GF as a culture substrate for neural progenitor cells (NPCs) and demonstrated that this scaffold material promotes the differentiation of BFCNs while maintaining excellent cell viability and proliferation.

Results: Immunofluorescence analysis, RT-PCR, western blotting and ELISA revealed that the efficiency of BFCN differentiation on 3D-GF was significantly greater than that on tissue culture polystyrene substrates. Furthermore, a cell adhesion study suggested that 3D-GF scaffold enhances the expression of adhesion proteins including vinculin, integrin and N-cadherin. These findings indicate that 3D-GF scaffold materials are excellent candidates for the differentiation of BFCNs from NPCs.

Conclusion: These results suggest new opportunities for the application of 3D-GF scaffold as a neural scaffold for Alzheimer's disease therapies based on NPCs.

Trial registration: Not applicable.

\section{Introduction}

Stem cell-based tissue engineering and regenerative medicine are at the forefront of current research in the treatment of various neurodegenerative diseases such as Alzheimer's disease (AD) $[1,2]$. AD patients present with an irreversible decline in cognitive function, resulting from damage to the forebrain cholinergic projection system, which innervates widespread regions of the cerebral cortex [3-5]. An early substantial loss of basal forebrain cholinergic neurons (BFCNs) is associated with $A D$ and deficits in spatial learning and memory in the central nervous system [6, 7]. Stem cells have been rapidly developed in numerous clinical cell-based therapies because of their self-renewal and differentiation abilities.

Recently, stem cell therapy for AD has been developed [2], but the most appropriate stem cell line has not been identified. In recent years, neural progenitor cells (NPCs) have been considered to be promising in cell-based $A D$ therapy, owing to their ability to differentiate into cholinergic neurons $[8,9]$.

The extracellular matrix (ECM) in cellular microenvironment plays an important role in cell behaviors [10]. For stem cell therapy, reconstructing the extracellular environment for stem cell differentiation is becoming increasingly attractive. The interaction between stem cells and the extracellular microenvironment is critical in controlling cell fate during stem cell differentiation [11]. To achieve the control of cell fate determination, many researchers have attempted to create three-dimensional (3D) 
materials that mimic the surface topography, mechanical properties, and chemical microenvironment of the ECM for use in tissue engineering and regenerative medicine [11]. These fields have experienced increasing research interest because of major progress in cell and organ transplantation [12-15]. Tissue engineering scaffolds would be required and should ideally provide suitable 3D microenvironments with desirable mechanical support for neural tissue regeneration [16].

Graphene, a single atomic monolayer of graphite, has attracted much interest for applications in medical field in the past several years [17-19]. Recently, 3D graphene foam (3D-GF) has been developed as scaffold materials for tissue engineering and regenerative medicine because it possesses high porosity, specific surface area and provides a 3D environment for cells [14, 20, 21]. 3D-GF has also been proposed as a neural scaffold for stem cell-based therapy, because 3D-GF with a 3D environment and surface wrinkles strongly induces the differentiation of NSCs to neuronal lineages [20]. In addition, 3D-GF has been demonstrated as a biodegradable scaffold with a two-stage mechanism for tissue engineering [14]. For this purpose, 3D-GF shows a great potential for the application in tissue engineering scaffolds and implantable medical devices. However, to the best of our knowledge, there are few reports regarding the ability of cells to differentiate into BFCNs in 3D extracellular environments.

In the present study, we investigated the capacity of NPCs to differentiate into BFCNs and cell adhesion ability utilizing 3D-GF as a cell scaffold. RT-PCR and western blotting were employed to detect the expression of choline acetyltransferase (ChAT) and a neurotrophin receptor (p75NTR, p75) in NPCs grown on 3D-GF by using polystyrene substrates (TCPS) as comparison. The acetylcholine (ACh) secretion was also studied. This work can validate the strong medical potential of the NPCs grown on functional 3D-GF scaffolds.

\section{Materials And Methods}

\subsection{Fabrication of the 3D-GF substrates}

3D-GF was synthesized by chemical vapor deposition (CVD) according to previous reports [22] by using a porous $\mathrm{Ni}$ foam as a growth template. After the metallic template was etched, the obtained samples were thoroughly rinsed with a solution of $\mathrm{HNO}_{3}$ or $\mathrm{HCl}$ and water to remove the trace amounts of catalyst and etching agents. 3D-GF was sterilized by soaking in $75 \%$ ethanol. Before the cells were seeded, the samples were soaked in cell culture medium overnight.

\subsection{Characterization of the graphene substrates}

The morphology of the graphene samples was characterized by scanning electron microscopy (SEM) (Quanta 400 FEG, FEI, USA). The crystallinity and number of layers within the graphene samples were examined by using Raman spectroscopy (lamRAM HR800, HORIBA, France) and transmission electron microscopy (TEM) (Tecnai G2 F20 S-Twin, FEI, USA).

\subsection{NPCs culture and differentiation assay}


NPCs were derived from the forebrains of mouse embryos at gestational day 14 (E14). All mice were housed and bred in the Comparative Medicine facilities of the University of Soochow in China. All procedures were conducted according to protocols approved by the Institutional Animal Care Committee. The forebrain of each E14 mouse embryo was dissected and dissociated into single cells by triturating the tissue with a $3 \mathrm{~mL}$ pipette. Cells from each forebrain were seeded into a $25 \mathrm{~cm}^{3}$ culture flask at a density of $2 \times 10^{5}$ cells $/ \mathrm{mL}$ in $10 \mathrm{~mL}$ of mouse NeuroCult NSC Proliferation Medium (Stem Cell, Canada) supplemented with epidermal growth factor $(20 \mathrm{ng} / \mathrm{mL})$ and basic fibroblast growth factor $(10 \mathrm{ng} / \mathrm{mL})$ for neurospheres culture. Neurospheres were passaged when reaching $100-150 \mathrm{~mm}$ in diameter. All cells were incubated at $37^{\circ} \mathrm{C}$ in a humidified incubator with $5 \% \mathrm{CO}_{2}$. For differentiation, in the first 24 hours, the medium was supplemented with $1 \%$ B-27 supplement, $100 \mathrm{ng} / \mathrm{mL}$ sonic hedgehog, $100 \mathrm{ng} / \mathrm{mL}$ fibroblast growth factor 8 , and $10 \mathrm{ng} / \mathrm{mL}$ bone morphogenetic protein-9 (BMP9). For the next 48 hours, the medium was supplemented with $10 \mathrm{ng} / \mathrm{mL}$ BMP9 and $1 \%$ B-27 supplement, after which the cells were never again exposed to BMP9. From D4 to D21, the medium was supplemented with $2 \%$ B-27, 2 mM glutamic acid, and $2.66 \mu \mathrm{M}$ arabinosylcytosine (AraC, to eliminate the growth of bFGF-responsive cells arising from fragments of undissociated neurospheres).

\subsection{SEM observation of NPCs cultured on 3D-GF and TCPS substrates}

NPCs grown on the two substrates were fixed with $2.5 \%$ glutaraldehyde in phosphate buffer (pH 7.4), for 1 hour at $4^{\circ} \mathrm{C}$, postfixed in $1 \%$ osmium tetroxide for 30 minutes at $4^{\circ} \mathrm{C}$, and dehydrated through a series of alcohol from $20-100 \%$. Finally, the attached cells were dried by lyophilization and coated with sputtered Au before examination with a Quanta $400 \mathrm{FEG} \mathrm{SEM} \mathrm{at} \mathrm{an} \mathrm{accelerating} \mathrm{voltage} \mathrm{of} 5 \mathrm{Kv}$. Each experiment was performed in triplicate ( $\mathrm{n}=5$ in each experiment).

\subsection{Cell viability}

NPCs were cultured on 3D-graphene and TCPS treated with the mouse NeuroCult NSC Proliferation Medium supplemented with epidermal growth factor $(20 \mathrm{ng} / \mathrm{mL})$ and basic fibroblast growth factor (10 $\mathrm{ng} / \mathrm{mL}$ ) for 7 days. The viability of NPCs was analyzed by using the LIVE/DEAD assay. In brief, $200 \mathrm{~mL}$ of the combined LIVE/DEAD cell staining solution ( $2 \mathrm{mM}$ calcein AM and $4 \mathrm{mM}$ EthD-1 in PBS) was added to each substrate and incubated with cells for $20 \mathrm{~min}$ at $37^{\circ} \mathrm{C}$. Images were obtained using an inverted fluorescence microscope (Eclipse Ti-E, Nikon, Japan). All assays were repeated three times (five wells per sample at each time point, $n=5$ ).

\subsection{Immunohistochemical staining}

The cells were treated according to the protocols of the Fast ImmunoCytoChemistry® Staining Kit (BPICC30-1KT, Protein Biotechnologies, USA). The primary antibodies used were as follows: anti-Nestin (Sigma, USA), anti-Ki67 (Abcam, USA), anti-ChAT (Millipore, Germany), anti-p75 (Millipore, Germany), antiMAP2 (Sigma, USA), anti-VAChT (Millipore, Germany), and anti-Synapsin 1 (Abcam, USA). The working 
concentration of DAPI (Sigma, USA) was $5 \mu \mathrm{g} / \mathrm{mL}$ in PBS. Each experiment was performed in triplicate ( $\mathrm{n}$ $=5$ in each experiment).

\subsection{Quantification of ACh in differentiation medium}

For quantification of $\mathrm{ACh}, 100 \mu \mathrm{L}$ of cell culture supernatant (day 21 of differentiation) was evaluated by using an AmplexRed Acetylcholine/Acetylcholinesterase Assay Kit (Invitrogen, USA) according to the manufacturer's protocol. In brief, ACh is monitored indirectly using 10-acetyl-3,7-dihydroxyphenoxazine (Amplex Red Reagent), a sensitive fluorogenic probe for $\mathrm{H}_{2} \mathrm{O}_{2}$ and an end product of oxidized choline. Fluorescence was monitored in a fluorescence plate reader using $550 \mathrm{~nm}$ for excitation and $595 \mathrm{~nm}$ for emission after $1 \mathrm{~h}$ of incubation with the reaction mix ( $\mathrm{n}=5$ for each experiment).

\subsection{Western blotting}

The differentiating cells were lysed in RIPA buffer (Beyotime, China) containing a complete protease inhibitor cocktail and $2 \mathrm{mM}$ phenylmethylsulfonyl fluoride. Approximately $20 \mu \mathrm{g}$ of total protein was separated by $10 \%$ sodium dodecyl sulfate polyacrylamide gel electrophoresis (SDS-PAGE) and then transferred to a $0.45-\mu \mathrm{m}$ nitrocellulose membrane (Millipore, USA). The protein concentrations were determined using a BCA Protein Assay Kit (Beyotime, China). The membranes were incubated with primary antibodies overnight at $4^{\circ} \mathrm{C}$ and then hybridized with the appropriate HRP-conjugated secondary antibody (Amersham Pharmacia Biotech, USA) for $2 \mathrm{~h}$ at room temperature. Protein signals were visualized using the ECL detection system. The primary antibodies were against ChAT (Millipore, Germany), p75 (Millipore, Germany), MAP2 (Sigma, USA), vinculin (Sigma, USA), integrin (Abcam, USA), $\mathrm{N}$-cadherin (Sigma, USA) and $\beta$-tubulin (Beyotime, China).

\subsection{Real-time polymerase chain reaction (RT-PCR)}

Total RNA and protein of differentiating NPCs (21 days) were extracted from NPCs cultured on 3D-GF scaffolds and TCPS substrates by using TRIzol reagent (Life Technologies, USA) according to the manufacturer's protocol. The mRNA was converted to cDNA in $20 \mu \mathrm{m}$ reaction volumes using a PrimeScriptTM RT Reagent Kit (Takara, Japan). Real-time PCR was performed using SYBR Premix Ex Taq TM (Takara, Japan), by following the manufacturer's instructions, and run on a 7500 Real-Time PCR system (Applied Biosystems, USA). The mRNAs were amplified using gene-specific PCR primer sequences for mouse, as shown in table 1 ( $n=5$ for each experiment).

\subsection{Statistical analysis}

All experiments were performed in triplicate and the data are shown as the means \pm SDs of three separate experiments. Statistical analysis was performed using one-way analysis of variance (ANOVA) followed by Tukey's test. Differences with $p<0.05$ were considered to be significant while $p<0.01$ was considered to be highly significant.

\section{Results}




\subsection{Biocompatibility of 3D-GF scaffold}

3D-GF scaffold was synthesized via the CVD method by using a Ni foam template, as described previously [20]. Ni was subsequently removed via $\mathrm{FeCl}_{3}$ etching. 3D-GF scaffolds were characterized by SEM and Raman spectroscopy, as shown in the supporting information (Figure S1). SEM images revealed a robust, 3D structure of 3D-GF and its interconnected pores, with a surface covered with many ripples and wrinkles. It can be observed that the pore of size 3D-GF was 100-300 $\mu \mathrm{m}$, whereas the width of the graphene skeleton was approximately 100-200 $\mu \mathrm{m}$. Furthermore, the shape of the 2D bands and the intensity ratio between the $2 \mathrm{D}$ and $\mathrm{G}$ bands demonstrated that 3D-GF was constructed from few-layer or multi-layer graphene sheets (Figure S1C). In addition, the graphene network possessed excellent mechanical strength and flexibility and free standing, allowing for cell growth in a 3D environment. NPCs were first incubated on 3D-GF scaffolds. For two days after cell seeding, in the proliferation medium, almost all cells adhered to the 3D-GF, and the interaction between NPCs and the 3D-GF was examined by SEM (Fig. 1A). NPCs cultured on 3D-GF exhibited excellent cell morphology, as well as cell density and adhesion similar to that observed on TCPS (Fig. 1C). The inset SEM image in Fig. 1A shows that the cells spread naturally, similarly to TCPS groups. Furthermore, 3D-GF cytotoxicity to NPCs was evaluated by using Calcein-AM and EthD-1 staining assays with TCPS as the control (Fig. 1B and 1D). Figure 1B and $1 \mathrm{E}$ show that almost all of the cells cultured on 3D-GF for 7 days were viable, and the difference in cell viability between 3D-GF and TCPS was insignificant. Those results suggest good biocompatibility of 3DGF and indicate that 3D-GF does not affect the cell viability, in agreement with results from previous reports $[17,18,20]$.

\subsection{Characterization of stemness and proliferation of NPCs on 3D-GF scaffold}

The cells were stained with an antibody against nestin, a protein marker of NPCs, to verify the stemness and authenticity of the NPCs. Figure 2A shows that nearly all of the cells on 3D-GF were immunopositive for nestin (red), and there were no obvious differences from the cells on TCPS. Furthermore, the percentage of nestin-positive cells (approximately $91.5 \%$ and $93.1 \%$ for 3D-GF and TCPS, respectively) indicated that on both substrates, almost all of the NPCs were stained for nestin, revealing that NPCs proliferated well on 3D-GF while maintaining their stemness. To address the proliferative effect of 3D-GF on NPCs, a proliferation assay was performed by measuring the expression of Ki67 protein, a cellular marker for NPC proliferation. Figure 2C and 2D show that a majority of cells (approximately $68.9 \%$ and $61.6 \%$, respectively) on 3D-GF and TCPS were stained positively for Ki67, thus indicating insignificant difference in proliferation between 3D-GF and TCPS. These results demonstrate that NPCs can grow well on this substrate and support the potential application of 3D-GF as a neural scaffold for NPC-based therapy in tissue engineering.

\subsection{Enhanced generation of BFCNs cultured on 3D-GF scaffold}


A previously described method [6] was applied to differentiate NPCs into BFCNs on 3D-GF. Phenotypic changes can be observed. After 21 days of differentiation culture, the cells exhibited an elongated cell shape on the 3D-GF surface (Fig. 3A). Immunofluorescence staining (Fig. 3A and 3B) shows that the differentiated NPCs were double-labeled for microtubule-associated protein 2 (MAP2, red) associated with mature neuron and ChAT (green) expressed by cholinergic neurons of both the basal forebrain and the motor system. The quantitative analysis (Fig. $3 \mathrm{C}$ ) suggests that approximately $30.5 \%$ of the neurons were ChAT positive on 3D-GF, whereas only approximately $9.7 \%$ of the cells on TCPS were ChAT positive neurons (Fig. 3C). Furthermore, the cholinergic neurons were positive for the mature neuronal markers p75 and vesicular acetylcholine transporter (VAChT) (Fig. 4A and 4B). p75 is a neurotrophin receptor and is also expressed by more than $95 \%$ of ChAT-positive cells in the basal forebrain [23]; VAChT is a glycoprotein responsible for the accumulation of acetylcholine into pre-synaptic vesicles in cholinergic neurons [24]. Thus, these results confirmed that NPCs cultured on 3D-GF differentiated into BFCNs. For a quantitative analysis, NPCs were harvested and subjected to RT-PCR and western blotting. RT-PCR analysis of the formed neurons after 21 day of differentiation (Fig. 4C) showed a significant increase in the expression of markers for the BFCN lineage, including MAP2, p75 and ChAT, compared to the TCPS groups. Meanwhile, as shown in Fig. 4D \& 4E, NPCs cultured on 3D-GF exhibited markedly higher expression of MAP2, p75 and ChAT than those in the TCPS groups with 2.8-, 2.0- and 2.5-fold, respectively. These results indicate that 3D-GF remarkably enhances the differentiation of NPCs into BFCNs.

\subsection{Functional identification of differentiated BFCNs on 3D- GF scaffold}

The function of the BFCNs was further verified by immunofluorescence staining for synapsin 1 and direct detection of ACh. Synapsin 1 is a marker specific for presynaptic terminals $[25,26]$. Staining for synapsin 1 showed a large number of presynaptic regions directly abutting neurite outgrowths from the differentiated cells on 3D-GF (Fig. 5A), thus suggesting that the BFCNs were initiating synaptic transmission and forming functional synapses with other neurons. RT-PCR analysis of synapsin 1 (Fig. 5B) confirmed a significant increase (approximately 9.7-fold compared with TCPS groups) in the expression of synapsin 1. Additionally, we detected ACh levels by using the AmplexRed Acetylcholine/Acetylcholinesterase Assay from the differentiation medium (Fig. 5C). The secretion of ACh by the differentiated cells on 3D-GF, approximately $4.72 \mu \mathrm{M}$, was significantly greater than that by the cells on TCPS, approximately $2.15 \mu \mathrm{M}$. This result also confirmed that neuronal ChAT immunopositivity was correlated with physiologically relevant ChAT enzymatic activity.

\subsection{Characterization of NPC adhesion}

To investigate the mechanism of the enhancing generation of BFCNs on 3D-GF, a quantitative analysis of proliferating NPCs on 3D-GF was performed to study the cell adhesion ability. The NPCs were harvested and subjected to RT-PCR and western blotting. The key proteins of cell adhesion including vinculin, integrin and $\mathrm{N}$-cadherin were detected. Vinculin is a component of adhesion plaques and adherens junctions in cells [27]; integrin is a type of cell adhesion protein that plays a critical role in the formation 
and remodeling of tissues and organs in multicellular organisms [28]; $\mathrm{N}$-cadherin belongs to a family of glycoproteins involved in $\mathrm{Ca}^{2+}$-dependent cell-cell adhesion [27, 29]. Figure 6A shows that NPCs cultured on 3D-GF exhibited significantly higher expression of vinculin, integrin and N-cadherin (approximately 4.7fold, 2.1-fold and 3.4-fold, respectively) than those in TCPS group. Furthermore, these increased expressions were re-validated by western blotting to assess the protein levels in the proliferating NPCs cultured on 3D-GF (Fig. 6B). These results demonstrate that 3D-GF enhances cell adhesion, thus potentially facilitating the differentiation of BFCNs from NPCs.

\section{Discussion}

In the central and peripheral nervous systems, ACh plays a significant role in synaptic transmission, where it mediates fast excitatory neurotransmission by binding to ACh receptors. Furthermore, ACh has been considered as a core neurotransmitter deficient in $A D$ in the cholinergic system [30,31]. AD, defined by progressive and irreversible cognitive decline [32], is the most common neurodegenerative disorder among elderly people. AD pathogenesis has been linked to a deficiency in the brain neurotransmitter ACh on the basis of findings that have correlated cholinergic system abnormalities with intellectual impairment [33]. The fundamental neuromorphological changes of $A D$ are severe loss of cholinergic neurons, widespread degeneration of cholinergic functions in the brain and a lack of ACh for controlling learning and memory in the central nervous system. Therefore, to develop therapies for $A D$, the number of $\mathrm{BFCNs}$ and the level of ACh should be enhanced through various means. Recent studies regarding treatments for $A D$ have mainly focused on augmenting residual cholinergic neurotransmission by using acetylcholinesterase inhibitors [32]. The development of stem cell-based therapy for AD has been proposed, with the main goals of enhancing the formation of BFCNs and the generation of ACh.

In this work, the experiments results demonstrate the enhanced generation of functional BFCNs from NPCs on 3D-GF: the proportion of BFCNs at 21 days of differentiation reached approximately $30.5 \%$ on 3D-GF compared with TCPS group that only presented $9.7 \%$. These BFCNs expressed all relevant markers of the BFCN lineage at both the mRNA and protein levels, produced ACh in vitro and also generated functional cholinergic synapses. In the present study, 3D-GF can promote the generation of BFCNs from NPCs, and the secretion of ACh by the differentiated cells grown on 3D-GF was approximately twice than that in the TCPS control groups. Our findings suggest that 3D-GF scaffold provide a potential platform for transplanted BFCNs to restore memory function in $A D$ because the increased ACh produced by BFCNs on 3D-GF can serve as a hippocampal neuromodulator and would play an important role in replacing the synapses lost in AD, thus enhancing memory function [6]. The properties of 3D-GF allow for various potential applications in the fields of tissue engineering and regenerative medicine. The results suggest that culturing NPCs on 3D-GF could provide an excellent source of cholinergic neurons for AD therapy.

To the best of our knowledge, it is the first study to apply 3D-GF for the generation of functional BFCNs. 3D-GF has been shown to provide positive cues for neuron differentiation $[18,20]$, which was further validated in the present work. Previous studies have demonstrated that the unique morphology of 3D-GF can promote the neuron differentiation. Thus, the notable difference between the generation of BFCNs on 
3D-GF versus TCPS substrates is probably due to the different surface morphology and spatial structure, given that the surface of 3D-GF structures possesses micro-scale topographic features different from those of TCPS (Figure S1). In particular, 3D-GF exhibits more complex 3D structures with interconnected pores, whereas TCPS has a relatively smooth surface with a 2D structure. All these factors may influence the behaviors of NPCs. Cell adhesion related to cell differentiation is essential for tissue engineering applications. Cell spreading, proliferation, and maintenance of cellular functions require good adhesion between the cells and scaffolds. In this work, we studied the cell adhesion ability by using western blotting and RT-PCR methods, and our results demonstrated that 3D-GF results in a significant enhancement in NPCs adhesion after 7 days of proliferation culture compared with TCPS. Therefore, the differential cell adhesion ability facilitates the enhancement of generating BFCNs on 3D-GF, which is in agreement with the studies indicating that the enhanced expression of adhesion proteins on graphene scaffolds facilitates cell differentiation $[11,34,35]$.

Although the differentiation data can be interpreted straightforwardly, challenges remain to be addressed before 3D-GF is used for cell transplantation in stem cell-based therapy in the future. First, it has been demonstrated that 3D-GF is biodegradable [14,34], but the degradation time is excessively long, thus requiring the acceleration of the degradation rate in future research. Second, the proportion of $\mathrm{ChAT}^{+} / \mathrm{MAP}^{+}{ }^{+}$cells should be increased. Third, it is unknown whether 3D-GF has the potential to enhance the generation of other functional neurons, such as dopaminergic neurons, serotoninergic neurons. Therefore, additional validation and future exploration may be required. Nonetheless, as a neural scaffold, 3D-GF facilitates the generation of neurons and shows great potential in the field of neural therapy.

\section{Conclusion}

This report describes the first utilization of 3D-GF as a neural scaffold for differentiation of NPCs in vitro. 3D-GF supports NPC growth, showing comparable biocompatibility and stemness to those of the commercial TCPS. Besides, compared to TCPS, 3D-GF greatly enhances the generation of functional BFCNs. After 21 days of differentiation culture, the proportion of BFCNs on 3D-GF was approximately three times than that on TCPS control substrates, thus suggesting that the topographical structures of the materials might affect the differentiation behaviors. Furthermore, the expression of adhesion proteins on 3D-GF showed a significant increase compared with the cells grown on TCPS; this increased cell adhesion mayinduce the increasing differentiation. The results presented here expand knowledge of the interactions between 3D-GF and BFCNs, and pave the way for future use of 3D-GF for tissue engineering, neural prostheses and other purposes.

\section{Abbreviations}

3D-GF three-dimensional graphene foam

NPCs neural progenitor cells 
AD Alzheimer's disease

ECM extracellular matrix

BFCNs basal forebrain cholinergic neurons

ChAT choline acetyltransferase

TCPS polystyrene substrates

ACh acetylcholine

CVD chemical vapor deposition

BMP9 bone morphogenetic protein-9

SEM scanning electron microscopy

TEM transmission electron microscopy

\section{Declarations}

\section{Ethics approval and consent to participate}

Not applicable.

\section{Consent for publication}

The current study is available from the corresponding author on reasonable request.

\section{Availability of data and material}

Data could be obtained upon request to the corresponding author.

\section{Funding}

This work was supported by grants from Guangdong Basic and Applied Basic Research Foundation (2019B1515120090), National Natural Science Foundation of China (51903246), and Natural Science Foundation of Jiangsu Province (BK20201196).

\section{Acknowledgment}


The authors thank M.S. Xiangxu Jiang from Soochow University for providing insightful discussions.

\section{Conflict of interest}

The authors have declared that there is no conflict of interest.

\section{Authors' contributions}

Concept or design: Ziyun Jiang, Sancheng Ma, Wei Jin and Guosheng Cheng

Acquisition of data: Ziyun Jiang, Lingyan Yang, Linhong Zhou and Miao Xiao

Analysis or interpretation of data: Ziyun Jiang, Lingyan Yang, Sancheng Ma, Wei Jin and Guosheng Cheng

Drafting of the manuscript: Ziyun Jiang, Lingyan Yang, Linhong Zhou, Miao Xiao, Sancheng Ma, Wei Jin and Guosheng Cheng

Critical revision of the manuscript for important intellectual content: Ziyun Jiang, Lingyan Yang, Linhong Zhou, Miao Xiao, Sancheng Ma, Wei Jin and Guosheng Cheng

All authors had full access to the data, contributed to the study, approved the final version for publication, and take responsibility for its accuracy and integrity.

\section{References}

1. Alrefai MT, Murali D, Paul A, Ridwan KM, Connell JM, Shum-Tim D. 2015 Cardiac tissue engineering and regeneration using cell-based therapy. Stem Cells Cloning. 8 81-101.

2. Yue $\mathrm{C}$, Jing $\mathrm{N}$. The promise of stem cells in the therapy of Alzheimer's disease. Transl Neurodegener. 2015;4:8.

3. Blurton-Jones M, Kitazawa M, Martinez-Coria H, Castello NA, Muller FJ, Loring JF, et al. 2009 Neural stem cells improve cognition via BDNF in a transgenic model of Alzheimer disease. Proc Natl Acad Sci U S A. 106 13594-13599.

4. Lee JK, Jin HK, Endo S, Schuchman EH, Carter JE, Bae JS. Intracerebral transplantation of bone marrow-derived mesenchymal stem cells reduces amyloid-beta deposition and rescues memory deficits in Alzheimer's disease mice by modulation of immune responses. Stem Cells. 2010;28:32943.

5. Liang J, Wu S, Zhao H, Li SL, Liu ZX, Wu J, et al. 2013 Human umbilical cord mesenchymal stem cells derived from Wharton's jelly differentiate into cholinergic-like neurons in vitro. Neurosci Lett. 532 $59-63$. 
6. Bissonnette CJ, Lyass L, Bhattacharyya BJ, Belmadani A, Miller RJ, Kessler JA. The controlled generation of functional basal forebrain cholinergic neurons from human embryonic stem cells. Stem Cells. 2011;29:802-11.

7. Hu Y, Qu ZY, Cao SY, Li Q, Ma L, Krencik R, et al. 2016 Directed differentiation of basal forebrain cholinergic neurons from human pluripotent stem cells. J Neurosci Methods. 266 42-49.

8. Antequera D, Portero A, Bolos M, Orive G, Hernandez RM, Pedraz JL, et al. 2012 Encapsulated VEGFsecreting cells enhance proliferation of neuronal progenitors in the hippocampus of AbetaPP/Ps1 mice. J Alzheimers Dis. 29 187-200.

9. Cusimano M, Biziato D, Brambilla E, Donega M, Alfaro-Cervello C, Snider S, et al. 2012 Transplanted neural stem/precursor cells instruct phagocytes and reduce secondary tissue damage in the injured spinal cord. Brain. 135 447-460.

10. Hu J, Seeberger PH, Yin J. Using carbohydrate-based biomaterials as scaffolds to control human stem cell fate. Org Biomol Chem. 2016;14:8648-58.

11. Chao TI, Xiang S, Chen CS, Chin WC, Nelson AJ, Wang C, et al. 2009 Carbon nanotubes promote neuron differentiation from human embryonic stem cells. Biochem Biophys Res Commun. 384 426430.

12. Langer R, Vacanti JP. Tissue engineering Science. 1993;260:920-6.

13. Mason C, Dunnill P. 2008 A brief definition of regenerative medicine. Regen Med. 3 1-5.

14. Loeblein M, Perry G, Tsang SH, Xiao W, Collard D, Coquet P, et al. 2016 Three-Dimensional Graphene: A Biocompatible and Biodegradable Scaffold with Enhanced Oxygenation. Adv Healthc Mater. 5 1177-1191.

15. Shadjou N, Hasanzadeh M. Graphene and its nanostructure derivatives for use in bone tissue engineering: Recent advances. J Biomed Mater Res A. 2016;104:1250-75.

16. Lalwani G, Gopalan A, D'Agati M, Sankaran JS, Judex S, Qin YX, et al. 2015 Porous three-dimensional carbon nanotube scaffolds for tissue engineering. J Biomed Mater Res A. 103 3212-3225.

17. Geim AK, Novoselov KS. The rise of graphene. Nat Mater. 2007;6:183-91.

18. Crowder SW, Prasai D, Rath R, Balikov DA, Bae H, Bolotin KI, et al. 2013 Three-dimensional graphene foams promote osteogenic differentiation of human mesenchymal stem cells. Nanoscale. 5 41714176.

19. Yang L, Tseng YT, Suo G, Chen L, Yu J, Chiu WJ, et al. 2015 Photothermal therapeutic response of cancer cells to aptamer-gold nanoparticle-hybridized graphene oxide under NIR illumination. ACS Appl Mater Interfaces. 7 5097-5106.

20. Li N, Zhang Q, Gao S, Song Q, Huang R, Wang L, et al. 2013 Three-dimensional graphene foam as a biocompatible and conductive scaffold for neural stem cells. Sci Rep. 31604.

21. Jiang Z, Song Q, Tang M, Yang L, Cheng Y, Zhang M, et al. 2016 Enhanced Migration of Neural Stem Cells by Microglia Grown on a Three-Dimensional Graphene Scaffold. ACS Appl Mater Interfaces. 8 25069-25077. 
22. Chen Z, Ren W, Gao L, Liu B, Pei S, Cheng HM. Three-dimensional flexible and conductive interconnected graphene networks grown by chemical vapour deposition. Nat Mater. 2011;10:424-8.

23. Mufson EJ, Ginsberg SD, Ikonomovic MD, DeKosky ST. Human cholinergic basal forebrain: chemoanatomy and neurologic dysfunction. J Chem Neuroanat. 2003;26:233-42.

24. Giboureau N, Som IM, Boucher-Arnold A, Guilloteau D, Kassiou M. 2010 PET radioligands for the vesicular acetylcholine transporter (VAChT). Curr Top Med Chem. 10 1569-1583.

25. Sudhof TC, Czernik AJ, Kao HT, Takei K, Johnston PA, Horiuchi A, et al. 1989 Synapsins: mosaics of shared and individual domains in a family of synaptic vesicle phosphoproteins. Science. $2451474-$ 1480.

26. Hedegaard C, Kjaer-Sorensen K, Madsen LB, Henriksen C, Momeni J, Bendixen C, et al. 2013 Porcine synapsin 1: SYN1 gene analysis and functional characterization of the promoter. FEBS Open Bio. 3 411-420.

27. Tozeren A, Wu S, Hoxter B, Xu W, Adamson ED, Byers SW. 1998 Vinculin and cell-cell adhesion. Cell Adhes Commun. 5 49-59.

28. Critchley DR, Holt MR, Barry ST, Priddle H, Hemmings L, Norman J. 1999 Integrin-mediated cell adhesion: the cytoskeletal connection. Biochem Soc Symp. 65 79-99.

29. Takeichi M. The cadherins: cell-cell adhesion molecules controlling animal morphogenesis. Development. 1988;102:639-55.

30. Mohr E, Mendis T, Rusk IN, Grimes JD. Neurotransmitter replacement therapy in Alzheimer's disease. J Psychiatry Neurosci. 1994;19:17-23.

31. Nirogi R, Mudigonda K, Kandikere V, Ponnamaneni R. Quantification of acetylcholine, an essential neurotransmitter, in brain microdialysis samples by liquid chromatography mass spectrometry. Biomed Chromatogr. 2010;24:39-48.

32. Gu G, Zhang W, Li M, Ni J, Wang P. Transplantation of NSC-derived cholinergic neuron-like cells improves cognitive function in APP/PS1 transgenic mice. Neuroscience. 2015;291:81-92.

33. Tabet N. Acetylcholinesterase inhibitors for Alzheimer's disease: anti-inflammatories in acetylcholine clothing! Age Ageing. 2006;35:336-8.

34. Nayak TR, Andersen H, Makam VS, Khaw C, Bae S, Xu X, et al. 2011 Graphene for controlled and accelerated osteogenic differentiation of human mesenchymal stem cells. ACS Nano. 5 4670-4678.

35. Ku SH, Lee M, Park CB. Carbon-based nanomaterials for tissue engineering. Adv Healthc Mater. 2013;2:244-60.

\section{Table}

Table 1 is not available with this version.

\section{Figures}



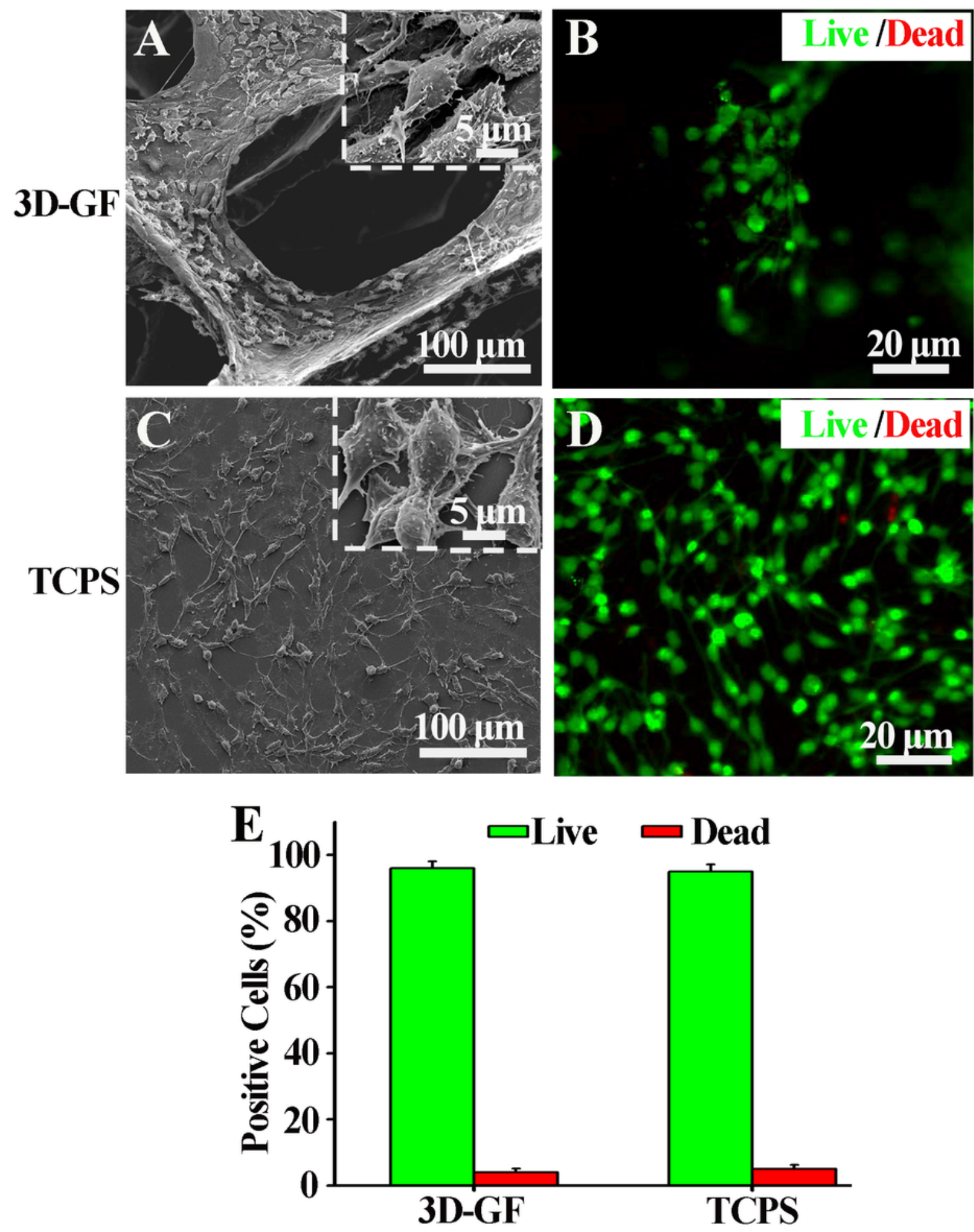

Figure 1

Assessment of the biocompatibility of 3D-GF toward NPCs. SEM images of NPCs cultured on 3D-GF (A) and TCPS (C) in the proliferation medium. The scale bar represents $100 \mu \mathrm{m}$. The inset shows a highmagnification SEM image of NPCs. The scale bar represents $5 \mu \mathrm{m}$. Cell viability assay of NPCs on 3D-GF (B) and TCPS (D) after 7 days of culture as determined by using the live/dead assay, in which live cells 
are stained green, and dead cells are stained red. The scale bar represents $20 \mu \mathrm{m}$. (E) The percentages of live cells on 3D-GF and TCPS.

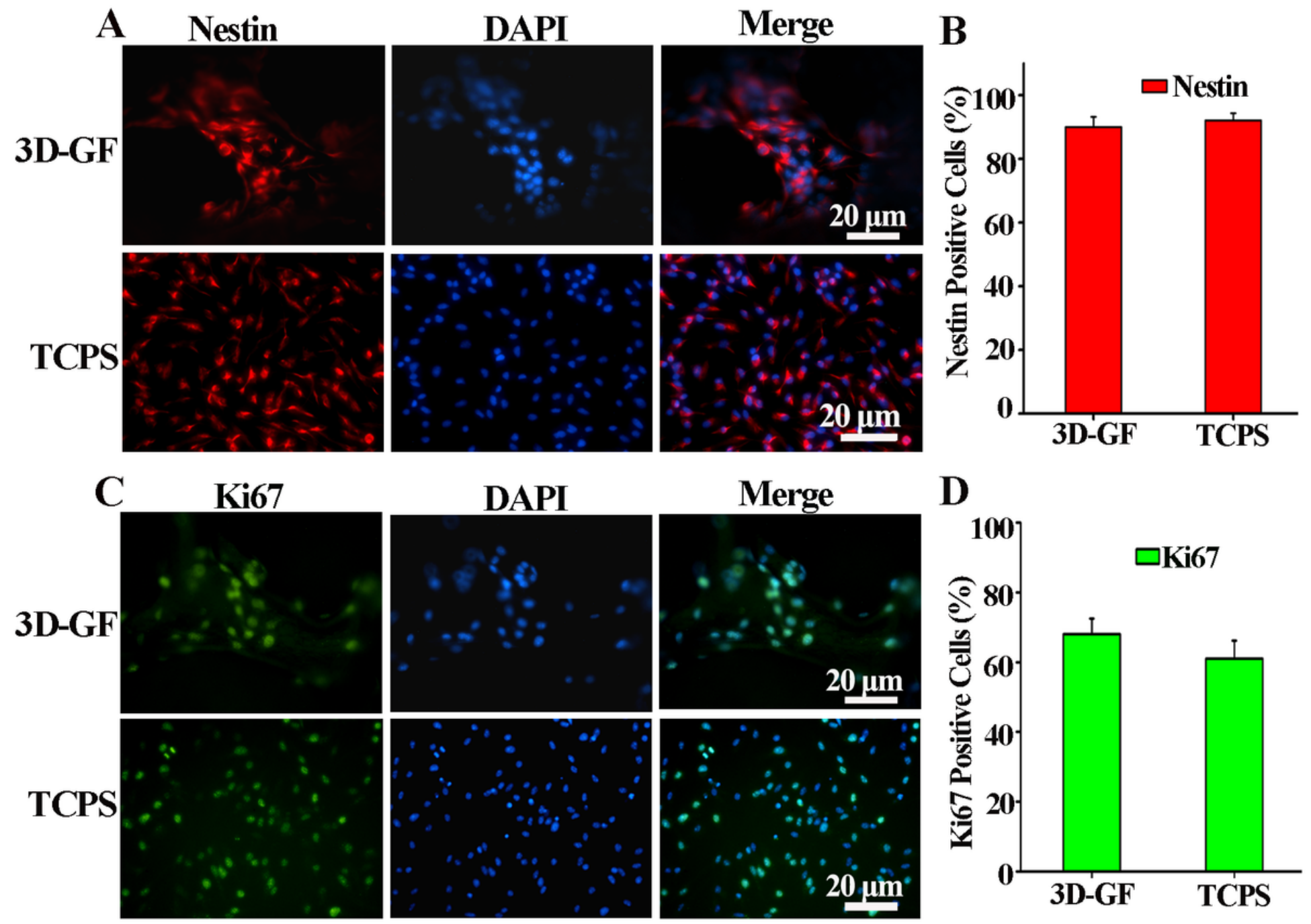

Figure 2

Stemness and proliferation assays of NPCs on 3D-GF. (A) Fluorescence images of NPCs grown on 3D-GF and TCPS for 7 days, stained with the markers nestin (red) for NPCs and DAPI (blue) for nuclei. The scale bar represents $20 \mu \mathrm{m}$. (B) The percentages of nestin-positive cells on 3D-GF and TCPS. (C) NSCs were double-stained with anti-Ki67 (green) and DAPI (blue) for nuclei. Ki67 is a marker for cell proliferation. The scale bar represents $20 \mu \mathrm{m}$. (D) The percentages of Ki67-positive cells on 3D-GF and TCPS. 

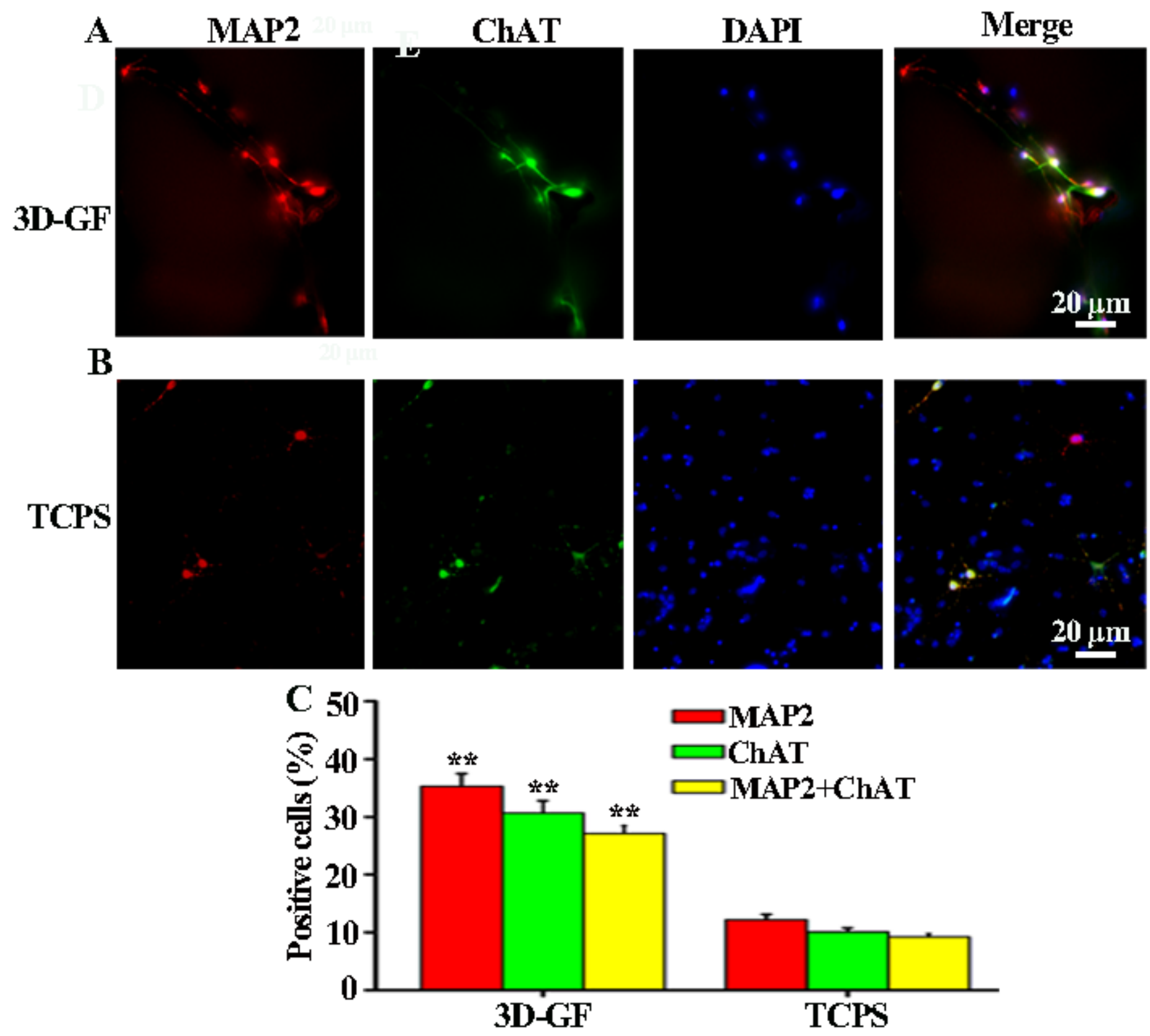

Figure 3

Enhanced generation of BFCNs on 3D-GF. Representative fluorescence images of differentiated NPCs after 21 days of differentiation culture on 3D-GF (A) and TCPS (B). The cells were immunostained for MAP2 (red) and ChAT (green) and stained with DAPI for nuclei (blue). The scale bar represents $20 \mu \mathrm{m}$. (C) Histograms showing the percentages of differentiated NPCs expressing MAP2 and ChAT. The data are presented as the means \pm standard deviations, ${ }^{\star \star} p<0.01$. 

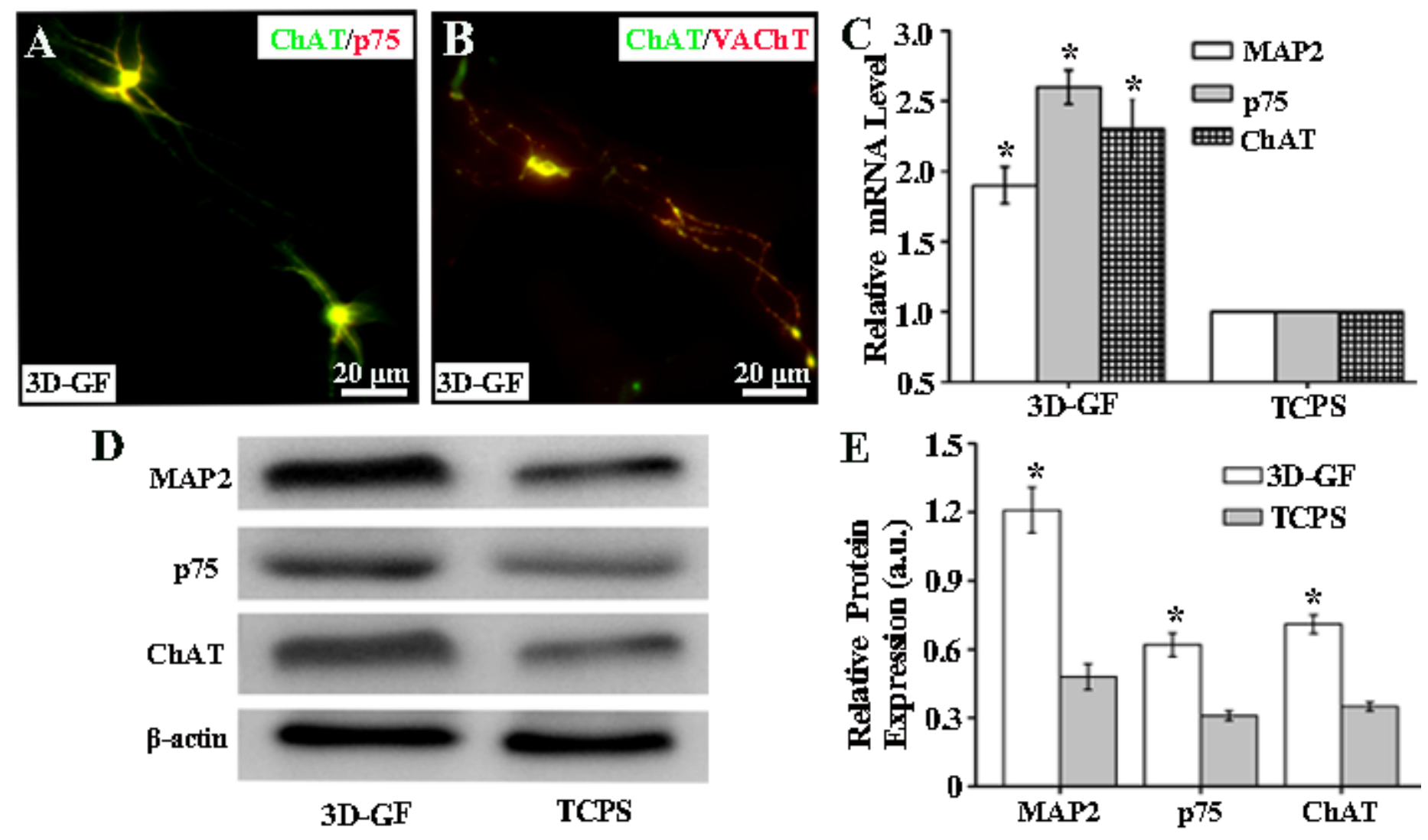

Figure 4

The quantitative analysis of mature neuronal markers of differentiated cells at Day 21. ChAT+ neurons expressing p75 (red, A) and VAChT (red, B). The scale bar represents $20 \mu \mathrm{m}$. (C) RT-PCR analysis showing 1.8- to 2.6-fold increases in the RNA levels for markers characteristic of the BFCNs on 3D-GF. (D) Western blot analysis of MAP2, p75 and ChAT protein expression levels in differentiated NPCs on 3D-GF and TCPS. (E) Relative optical densities of MAP2, p75 and ChAT bands shown in (D). The data are presented as the means \pm standard deviations, ${ }^{*} \mathrm{p}<0.05$. 

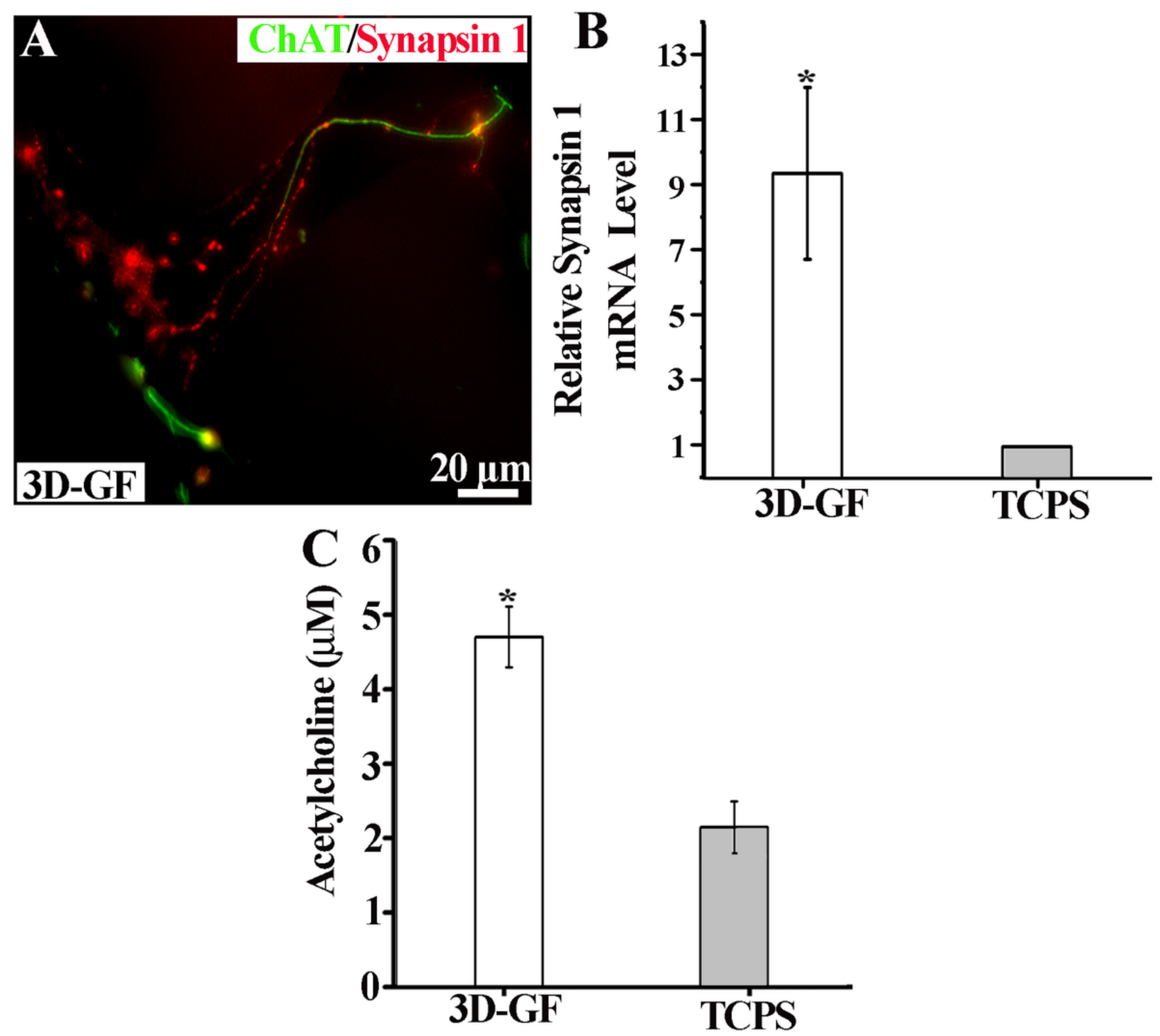

Figure 5

3D-GF facilitates the functionality of BFCNs. (A) The cells were immunostained for synapsin 1 (red) and ChAT (green). The scale bar represents $20 \mu \mathrm{m}$. (B) RT-PCR analysis shows a 9.7-fold increase in the RNA level of synapsin 1 in BFCNs on 3D-GF. (C) The secretion of ACh by BFCNs on 3D-GF and TCPS. The data are presented as the means \pm standard deviations, ${ }^{*} p<0.05$. 


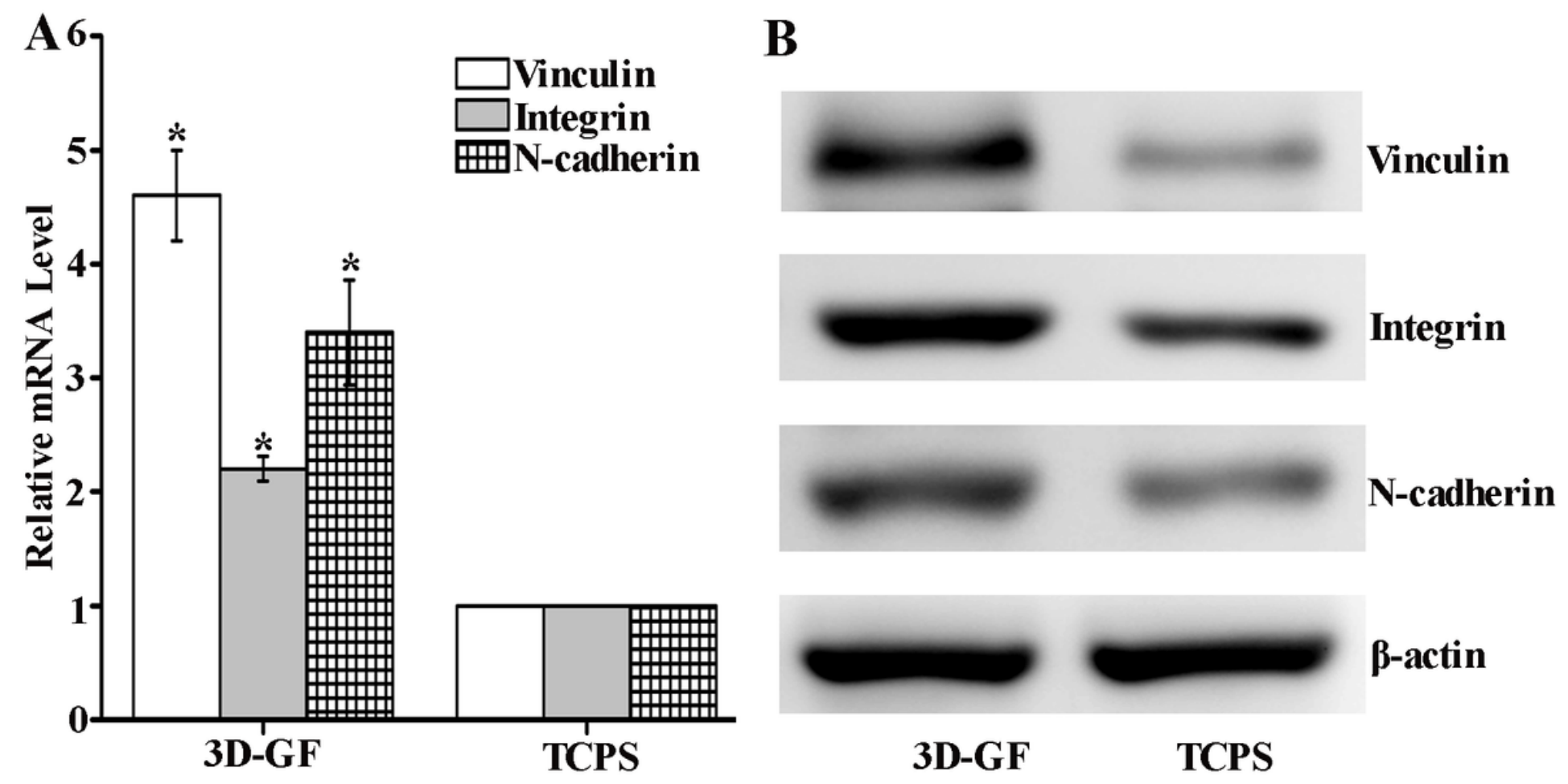

Figure 6

3D-GF promotes NPC adhesion. (A) mRNA expression levels of NPCs cultured on 3D-GF and TCPS, showing the ability of NPCs to adhere to the substrate. Gene expression of vinculin, integrin and Ncadherin was significantly increased on 3D-GF compared with TCPS. (B) Western blot analysis of vinculin, integrin and N-cadherin protein expression in proliferating NPCs cultured on 3D-GF and TCPS for 7 days. The data are presented as the means \pm standard deviations, ${ }^{*} p<0.05$. 